\title{
FCM Regimen
}

National Cancer Institute

\section{Source}

National Cancer Institute. FCM Regimen. NCI Thesaurus. Code C140730.

A regimen consisting of fludarabine, cyclophosphamide and mitoxantrone that can be used in the treatment of non-Hodgkin lymphoma (NHL). 